\title{
DOENÇA DE CROHN E CÁLCULO RENAL: muito mais que coincidência?
}

\author{
Maria Lenise Lopes VIANA, Rose Meire Albuquerque PONTES, Waldir Eduardo GARCIA, \\ Maria Emília FÁVERO, Denise Cavenaghi PRETE e Tiemi MATSUO
}

RESUMO - Racional - A doença de Crohn é uma doença inflamatória intestinal que pode estar associada a várias complicações e manifestações que são secundárias ao processo inflamatório de base. Em aproximadamente 30\% dos pacientes têm sido encontradas manifestações extraintestinais. A nefrolitíase é uma delas e a formação de cálculos é mais comum nestes pacientes do que na população em geral, principalmente cálculos de oxalato de cálcio. Objetivos - Avaliar os fatores metabólicos urinários potencialmente envolvidos na formação de cálculos renais em pacientes com doença de Crohn. Métodos - Foram avaliados 29 pacientes com doença de Crohn atendidos no Ambulatório de Doenças Inflamatórias do Hospital de Clínicas da Universidade Estadual de Londrina, PR, no período de janeiro a dezembro de 2004. A avaliação metabólica incluiu medidas séricas e urinárias de substâncias relacionadas à litíase renal, ultra-sonografia de rins e vias urinárias e cálculo da supersaturação urinária para o oxalato de cálcio, ácido úrico e fosfato de cálcio. Resultados - Dos 29 pacientes avaliados, 65,5\% eram do sexo feminino e 34,5\% do masculino. As principais alterações metabólicas urinárias encontradas foram 72,41\% de hipocitratúria, 41,4\% de hipomagnesiúria, 13,6\% de hiperoxalúria e 17,24\% de baixo volume urinário. Foram encontrados cálculos renais em 13 pacientes (44,8\%). Os pacientes submetidos a cirurgia intestinal, com ressecção ileal, apresentaram alterações na saturação urinária de oxalato e fosfato. Para a comparação das médias, utilizou-se o teste de Mann-Whitney e para determinar a associação entre as variáveis foi utilizado o teste do Qui-quadrado ou o teste exato de Fisher com um nível de significância de 5\%. Conclusão - A freqüência de pacientes com cálculos renais foi maior que a encontrada na população geral e acima do descrito em trabalhos similares. As alterações encontradas como hipocitratúria e a hipomagnesiúria, que representam fatores de risco reconhecido para a formação de cálculos, devem ter participação ativa nestes resultados. O impacto dessas alterações, medido pela saturação urinária elevada de oxalato e fosfato de cálcio, representa uma das maneiras de se demonstrar como estes fatores predispõem à nucleação de cristais e, conseqüentemente, à formação de cálculos nas vias urinárias.

DESCRITORES - Doença de Crohn. Cálculos renais. Oxalato de cálcio.

\section{INTRODUÇÃO}

A formação de cálculos renais em pacientes com doença inflamatória intestinal é mais comum do que na população geral. A freqüência de litíase neste grupo de pacientes varia de $12 \%$ a $28 \%{ }^{(10)}$, enquanto que na população em geral afeta de $1 \%$ a $14 \%$ das pessoas ${ }^{(6)}$. Vários fatores têm sido associados ao seu aparecimento, entre eles o volume urinário, $\mathrm{pH}$, excreção de oxalato, citrato e magnésio, embora não se tenha chegado a consenso sobre o grau de importância de cada um destes fatores litogênicos ${ }^{(2,5,9,12,15,18,19)}$.

$\mathrm{Na}$ doença de Crohn, a maioria dos cálculos é constituída de oxalato de cálcio, devido a maior absorção e excreção urinária de oxalato. $\mathrm{O}$ oxalato proveniente da dieta, normalmente, não é absorvido quando ligado ao cálcio no intestino, porém nos casos de má absorção ou ressecção cirúrgica do intestino, permanece livre devido à fixação do cálcio aos ácidos graxos. Pode, também, ocorrer diminuição da quantidade de Oxalobacter formigens, uma bactéria intestinal responsável pela degradação do oxalato. Ademais, os ácidos biliares e os próprios ácidos graxos aumentam a permeabilidade do cólon ao oxalato. $\mathrm{O}$ conjunto dos fatores já mencionados favorece o aparecimento de hiperoxalúria ${ }^{(8,11,14)}$.

A formação de cálculos no trato urinário é um processo multifatorial que envolve o balanço entre os fatores promotores da formação de cálculo e os inibidores da cristalização ${ }^{(7,17)}$.

O propósito deste estudo foi analisar os principais fatores promotores da litogênese (cálcio, oxalato, fosfato e ácido úrico), fatores inibidores (citrato, magnésio) e o grau de supersaturação urinária em relação ao oxalato de cálcio, ácido úrico e fosfato de cálcio. Acredita-se que a compreensão desses fatores seja útil na prevenção do aparecimento e da recurrência da nefrolitíase nos pacientes com doença de Crohn, condição por si só bastante agressiva e que desencadeia alterações importantes na vida das pessoas por ela acometidas.

\section{MÉTODOS}

Após aprovação pelo Comitê de Ética do Hospital Universitário da Universidade Estadual de Londrina

Departamento de Clínica Médica, Centro de Ciências da Saúde, Universidade Estadual de Londrina, PR.

Correspondência: Dra. Maria Lenise Lopes Viana - Rua Madre Leonia Milito, 102 - Jd. Bela Suíça - 86050-270 - Londrina, PR. E-mail: maria.viana@unopar.br 
(Parecer CEP 048/03), foram incluídos no estudo 29 pacientes com doença de Crohn, com idade de 17 a 79 anos (40,2 \pm 13,1), sendo 18 mulheres. Esses pacientes eram acompanhados no Ambulatório de Doenças Inflamatórias Intestinais e apresentavam acometimento de intestino delgado isolado ou delgado e cólon, simultaneamente. O diagnóstico de doença de Crohn foi estabelecido por meio de critérios reconhecidos e aceitos internacionalmente ${ }^{(3)}$. As seguintes condições foram excluídas: pacientes com disfunção renal (creatinina sérica maior que $1,5 \mathrm{mg} / \mathrm{dL}$ ) e hipertireoidismo. A coleta das amostras de urina foi realizada na fase inativa da doença, determinada por CDAI (Crohn's Disease Activity Index) menor que $150^{(4)}$ e na ausência de infecção urinária. Foi verificada a utilização de medicamentos que, sabidamente, poderiam interferir nos resultados bioquímicos dos parâmetros analisados ${ }^{(1)}$.

A avaliação metabólica incluiu medidas séricas e urinárias, ultra-sonografia de rins e vias urinárias e determinação do índice de saturação urinária em relação ao oxalato de cálcio, ácido úrico e fosfato de cálcio. No sangue dos pacientes em jejum foram dosados creatinina, ácido úrico, cálcio e magnésio e gasometria venosa para medida de $\mathrm{pH}$ e bicarbonato.

Em urina de 24 horas (duas amostras) foram dosados oxalato, citrato, magnésio, fosfato, sódio, potássio, cloreto e volume. A análise do sedimento urinário e a medida de $\mathrm{pH}$ foram realizadas em amostra isolada de urina. Os pacientes foram cuidadosamente instruídos como proceder à coleta de urina de 24 horas, que foi colhida em galão plástico sem conservante.

O cálcio foi determinado por espectrofotometria de absorção atômica, o ácido úrico pelo método da uricase, a creatinina pelo método de Jaffé, o fosfato pelo método do fosfomolibdato, o magnésio pelo método azul de metiltimol, o sódio, potássio e cloreto foram determinados através de eletrodo de íon seletivo. $\mathrm{O}$ citrato foi dosado por método enzimático, em urina acidificada, utilizando citrato lyase. O oxalato foi dosado em urina acidificada através de método indireto por determinação do cálcio contido no precipitado de oxalato de cálcio, induzido na amostra ${ }^{(16)}$. O pH urinário foi medido em amostra isolada através de determinação potenciométrica em pHmetro Celm.

O exame ultra-sonográfico foi realizado por um mesmo observador com aparelho Voluson $730 \mathrm{GE}$, transdutor de 2 a $5 \mathrm{MHZ}$ para verificar a presença de cálculo renal e avaliar a morfologia do trato urinário.

$\mathrm{O}$ índice de saturação urinária foi calculado utilizando-se do software EQUIL-93.

Foi realizada a análise estatística descritiva e os dados identificados com média, desvio padrão, valores mínimo e máximo, mediana e freqüências relativa e absoluta. Para comparação das medianas utilizou-se teste U de Mann-Whitney. Para determinar associação entre as variáveis foi utilizado teste do Qui-quadrado ou o teste Exato de Fisher. O nível de significância adotado foi de $5 \%$ em todos os testes.

\section{RESULTADOS}

Dos 29 pacientes avaliados, o segmento ileal encontrava-se acometido em 55,2\% deles. Em 48\% o diagnóstico da doença era recente (menos de 5 anos) e 65,5\% deles já haviam sido submetidos a procedimento cirúrgico em região intestinal, sendo que em 57,9\% desses houve remoção de algum segmento ileal. Apenas dois pacientes não apresentaram nenhum parâmetro urinário considerado como potencial distúrbio metabólico associado à nefrolitíase. Três pacientes apresentaram somente um distúrbio e 24, dois ou mais distúrbios urinários litogênicos (Figura 1). Todos tinham função renal normal com níveis de creatinina de $0,8 \pm 0,2 \mathrm{mg} / \mathrm{dL}$. Seis mostraram $\mathrm{pH}$ urinário abaixo de 5,5, mas esse parâmetro isoladamente não foi considerado fator potencial para a litogênese, uma vez que os pacientes não revelaram supersaturação para ácido úrico. A Tabela 1 mostra a freqüência das alterações metabólicas urinárias, sendo a hipocitratúria $(72,4 \%)$ e a hipomagnesiúria $(48,3 \%)$ os achados mais freqüentes. Nenhum paciente desenvolveu hiperfosfatúria. Dos 21 que apresentaram hipocitratúria $(72,4 \%), 8$ possuiam ressecção ileal. Dos 14 pacientes com hipomagnesiúria $(48,3 \%)$ somente 1 não mostrou hipocitratúria associada e 6 deles possuiam ressecção ileal.

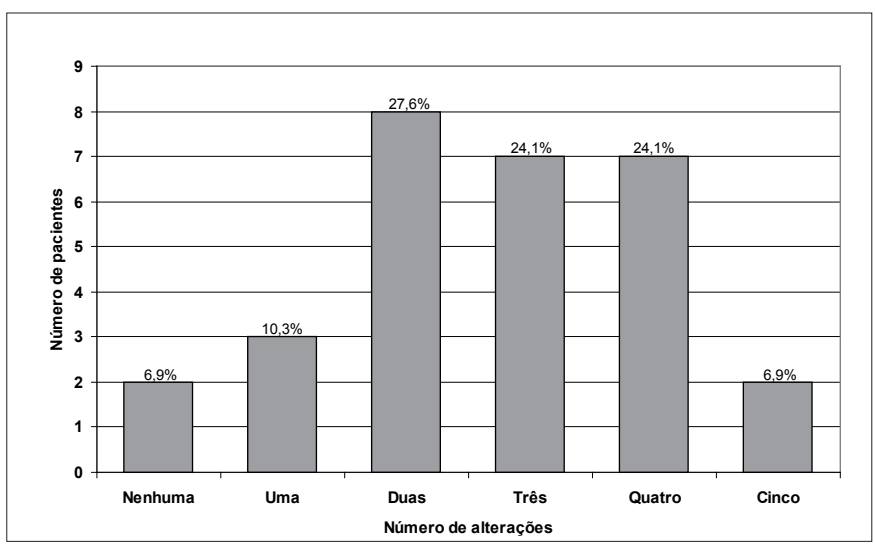

FIGURA 1. Percentagem de alterações metabólicas nas amostras de urina de 24 horas dos pacientes com doença de Crohn

TABELA 1. Freqüência das alterações metabólicas urinárias potencialmente ligadas à litogênese

\begin{tabular}{lcc}
\hline & $\mathrm{n}$ & $\%$ \\
\hline Volume urinário baixo $<15 \mathrm{~mL} / \mathrm{kg} / 24 \mathrm{~h}$ & 5 & 17,2 \\
Ácido úrico $>750 \mathrm{mg} / 24 \mathrm{~h}$ & 1 & 3,4 \\
Cálcio $>4 \mathrm{mg} / \mathrm{kg} / 24 \mathrm{~h}$ & 1 & 3,4 \\
Magnésio $<50 \mathrm{mg} / 24 \mathrm{~h}$ & 14 & 48,3 \\
Sódio $>170 \mathrm{mEq} / 24 \mathrm{~h}$ & 13 & 44,8 \\
Citrato $<320 \mathrm{mg} / 24 \mathrm{~h}$ & 21 & 72,4 \\
Oxalato $>40 \mathrm{mg} / 24 \mathrm{~h}$ & 4 & 13,6 \\
\hline
\end{tabular}

A análise ultra-sonográfica detectou a presença de cálculo renal em 13 pacientes $(44,8 \%)$. Nove eram do sexo feminino $(69,2 \%)$ e quatro do masculino $(30,8 \%)$, com média de idade de 37,8 anos. Destes, cinco apresentaram hipocitratúria isolada, três, hipocitratúria associada com hipomagnesiúria, um apresentou hiperoxalúria, hipocitratúria e hipomagnesiúria, dois, baixo volume urinário, hipocitratúria e hipomagnesiúria, um, hipocitratúria e 
hipomagnesiúria e apenas um deles não apresentou nenhuma alteração metabólica urinária.

Quando se compararam os parâmetros urinários dos pacientes operados e não operados, verificou-se que nos primeiros (sobretudo naqueles com ressecção do íleo), a excreção dos fatores inibidores (citrato e magnésio) foi menor e dos fatores promotores (cálcio, oxalato, ácido úrico, fósforo e sódio), maior que nos não operados. O oxalato urinário foi significativamente maior nos pacientes com cirurgia intestinal $(28,4 \pm 17,5)$ do que naqueles sem antecedentes de cirurgia $(16,4 \pm 11,0)$. Nos pacientes que haviam sido submetidos a ressecção de algum segmento ileal, a excreção de oxalato urinário também foi maior $(35,3 \pm 19,2)$ do que naqueles que possuíam segmento ileal intacto $(17,6 \pm 10,1)(P<0,05)$. A excreção urinária de fósforo também foi maior nos pacientes com ressecção ileal $(612,3 \pm 192,0)$ em comparação com os com íleo intato $(442,8 \pm 226,7)(P<0,05)$. Nos pacientes com cálculo renal não se observou diferença estatisticamente significante para os fatores metabólicos em relação aos não possuidores de cálculo (Tabela 2).
A Tabela 3 mostra a supersaturação relativa de oxalato de cálcio, fosfato de cálcio e ácido úrico. Os pacientes com cirurgia intestinal apresentaram urina supersaturada para oxalato de cálcio e fosfato de cálcio ( $P=0,007$ e $P=0,015$, respectivamente), assim como aqueles com ressecção ileal $(P=0,007$ e $P=0,019)$. Não se observou associação entre a presença de cirurgia intestinal, de ressecção ileal e supersaturação urinária em relação aos pacientes com cálculo renal.

\section{DISCUSSÃO}

Em pacientes com doença de Crohn, o aumento da excreção urinária de oxalato tem sido descrito como o principal fator de risco litogênico em decorrência da hiperoxalúria associada à síndrome de má absorção vivenciada por $\operatorname{eles}^{(5,10,12)}$.

Apesar dos pacientes deste estudo não apresentarem grandes alterações na excreção de oxalato, quando se comparam os pacientes submetidos a cirurgia intestinal com não operados, observa-se diferença com significância estatística $(P<0,05)$ entre os grupos. Quando esses pacientes submetidos a cirurgia

TABELA 2. Distribuição dos pacientes submetidos a cirurgia intestinal, com ressecção ileal e com cálculo renal segundo a excreção média dos solutos urinários de 24 horas dos pacientes com doença de Crohn

\begin{tabular}{|c|c|c|c|c|c|c|c|c|c|}
\hline \multirow{2}{*}{$\begin{array}{l}\text { Análises } \\
\text { urinárias }\end{array}$} & \multicolumn{2}{|c|}{ Cirurgia intestinal } & \multirow[t]{2}{*}{ Valor de $P^{*}$} & \multicolumn{2}{|c|}{ Ressecção ileal } & \multirow[t]{2}{*}{ Valor de $P^{*}$} & \multicolumn{2}{|c|}{ Cálculo renal } & \multirow[t]{2}{*}{ Valor de $P^{*}$} \\
\hline & $\operatorname{Sim}(19)$ & Não (10) & & $\operatorname{Sim}(11)$ & Não (18) & & $\operatorname{Sim}(13)$ & Não (16) & \\
\hline $\begin{array}{l}\text { Volume urinário } \\
(\mathrm{mL} / 24 \mathrm{~h})\end{array}$ & $\begin{array}{c}1315,0 \pm 547,3 \\
(535,0-700,0) \\
1400,0^{\dagger}\end{array}$ & $\begin{array}{c}1216,5 \pm 283,8 \\
(510,0-495,0) \\
1322,5^{\dagger}\end{array}$ & 0,551 & $\begin{array}{c}1309,1 \pm 627,4 \\
(640,0-700,0) \\
1220,0^{\dagger}\end{array}$ & $\begin{array}{c}1264,2 \pm 362,2 \\
(510,0-2090,0) \\
1350,0^{\dagger}\end{array}$ & 0,964 & $\begin{array}{c}1300,0 \pm 577,4 \\
(510,0-2700,0) \\
1350,0^{\dagger}\end{array}$ & $\begin{array}{c}1265,9 \pm 380,5 \\
(640,0-2090,0) \\
1325,0^{\dagger}\end{array}$ & 0,759 \\
\hline PH urinário & $\begin{array}{c}6,1 \pm 0,5 \\
(5,4-7,1) \\
6,1^{\dagger}\end{array}$ & $\begin{array}{c}5,8 \pm 0,4 \\
(5,3-6,5) \\
5,8^{\dagger}\end{array}$ & 0,073 & $\begin{array}{c}6,2 \pm 0,5 \\
(5,4-7,1) \\
6,0^{\dagger}\end{array}$ & $\begin{array}{c}5,9 \pm 0,5 \\
(5,3-7,1) \\
5,9^{\dagger}\end{array}$ & 0,110 & $\begin{array}{c}6,0 \pm 0,5 \\
(5,4-7,1) \\
6,0^{\dagger}\end{array}$ & $\begin{array}{c}6,0 \pm 0,5 \\
(5,3-7,1) \\
5,9^{\dagger}\end{array}$ & 0,843 \\
\hline $\begin{array}{l}\text { Oxalato } \\
(\mathrm{mg} / 24 \mathrm{~h})\end{array}$ & $\begin{array}{c}28,4 \pm 17,5 \\
(10,8-79,7) \\
22,3^{\dagger}\end{array}$ & $\begin{array}{c}16,4 \pm 11,0 \\
(8,0-42,5) \\
13,0^{\dagger}\end{array}$ & $0,022^{\ddagger}$ & $\begin{array}{c}35,3 \pm 19,2 \\
(17,1-79,7) \\
31,0^{\dagger}\end{array}$ & $\begin{array}{c}17,6 \pm 10,1 \\
(8,0-42,5) \\
14,9^{\dagger}\end{array}$ & $0,002^{\ddagger}$ & $\begin{array}{c}23,1 \pm 15,6 \\
(8,0-57,6) \\
17,1^{\dagger}\end{array}$ & $\begin{array}{c}25,2 \pm 17,5 \\
(8,5-79,7) \\
20,7^{\dagger}\end{array}$ & 0,497 \\
\hline $\begin{array}{l}\text { Cálcio } \\
\text { (mg/24h) }\end{array}$ & $\begin{array}{c}96,8 \pm 56,0 \\
(41,8-255,9) \\
70,4^{\dagger}\end{array}$ & $\begin{array}{c}79,6 \pm 30,8 \\
(38,3-139,4) \\
79,4^{\dagger}\end{array}$ & 0,646 & $\begin{array}{c}140,7 \pm 66,6 \\
(43,6-255,9) \\
90,5^{\dagger}\end{array}$ & $\begin{array}{c}82,4 \pm 33,7 \\
(38,3-166,2) \\
73,2^{\dagger}\end{array}$ & 0,529 & $\begin{array}{c}97,3 \pm 55,7 \\
(38,3-255,9) \\
88,9^{\dagger}\end{array}$ & $\begin{array}{c}85,6 \pm 43,8 \\
(41,5-185,5) \\
72,4^{\dagger}\end{array}$ & 0,568 \\
\hline $\begin{array}{l}\text { Fósforo } \\
\text { (mg/24h) }\end{array}$ & $\begin{array}{c}530,3 \pm 202,0 \\
(195,0-860,0) \\
530,0^{\dagger}\end{array}$ & $\begin{array}{c}463,0 \pm 273,6 \\
(50,0-900,0) \\
400,0^{\dagger}\end{array}$ & 0,409 & $\begin{array}{c}612,3 \pm 192,0 \\
(310,0-860,0) \\
665,0^{\dagger}\end{array}$ & $\begin{array}{c}442,8 \pm 226,7 \\
(50,0-900,0) \\
410,0^{\dagger}\end{array}$ & $0,034^{\ddagger}$ & $\begin{array}{c}456,9 \pm 273,9 \\
(50,0-900,0) \\
370,0^{\dagger}\end{array}$ & $\begin{array}{c}547,8 \pm 178,6 \\
(285,0-795,0) \\
547,5^{\dagger}\end{array}$ & 0,203 \\
\hline $\begin{array}{l}\text { Ácido úrico } \\
(\mathrm{mg} / 24 \mathrm{~h})\end{array}$ & $\begin{array}{c}499,6 \pm 121,7 \\
(305,8-745,6) \\
470,4^{\dagger}\end{array}$ & $\begin{array}{c}387,0 \pm 212,6 \\
(109,9-764,2) \\
412,3^{\dagger}\end{array}$ & 0,130 & $\begin{array}{c}511,4 \pm 122,2 \\
(318,8-745,6) \\
470,4^{\dagger}\end{array}$ & $\begin{array}{c}430,0 \pm 181,8 \\
(110,0-764,1) \\
445,5^{\dagger}\end{array}$ & 0,243 & $\begin{array}{c}412,5 \pm 156,7 \\
(109,9-745,6) \\
420,1^{\dagger}\end{array}$ & $\begin{array}{c}500,3 \pm 164,6 \\
(119,1-764,2) \\
497,6^{\dagger}\end{array}$ & 0,065 \\
\hline $\begin{array}{l}\text { Magnésio } \\
(\mathrm{mg} / 24 \mathrm{~h})\end{array}$ & $\begin{array}{c}50,4 \pm 22,8 \\
(12,8-107,0) \\
47,9^{\dagger}\end{array}$ & $\begin{array}{c}63,1 \pm 36,9 \\
(14,6-122,8) \\
55,5^{\dagger}\end{array}$ & 0,463 & $\begin{array}{c}50,8 \pm 26,9 \\
(12,8-107,1) \\
42,0^{\dagger}\end{array}$ & $\begin{array}{c}57,2 \pm 30,0 \\
(14,6-122,8) \\
56,5^{\dagger}\end{array}$ & 0,345 & $\begin{array}{c}57,9 \pm 33,0 \\
(14,6-122,8) \\
49,9^{\dagger}\end{array}$ & $\begin{array}{c}52,3 \pm 25,1 \\
(12,8-114,5) \\
51,4^{\dagger}\end{array}$ & 0,726 \\
\hline $\begin{array}{l}\text { Citrato } \\
(\mathrm{mg} / 24 \mathrm{~h})\end{array}$ & $\begin{array}{c}214,9 \pm 171,9 \\
(32,4-638,8) \\
204,0^{\dagger}\end{array}$ & $\begin{array}{c}291,3 \pm 300,2 \\
(39,5-1073,4) \\
216,9^{\dagger}\end{array}$ & 0,582 & $\begin{array}{c}208,1 \pm 173,8 \\
(35,4-639,8) \\
204,1^{\dagger}\end{array}$ & $\begin{array}{c}261,5 \pm 249,7 \\
(32,4-1073,5) \\
193,9^{\dagger}\end{array}$ & 0,686 & $\begin{array}{c}255,8 \pm 296,1 \\
(35,4-1073,4) \\
204,0^{\dagger}\end{array}$ & $\begin{array}{c}229,4 \pm 147,1 \\
(32,4-476,5) \\
193,9^{\dagger}\end{array}$ & 0,511 \\
\hline
\end{tabular}


TABELA 3. Distribuição dos pacientes submetidos a cirurgia intestinal, com ressecção ileal e com cálculo renal segundo a supersaturação urinária média de oxalato de cálcio, brushita e ácido úrico

\begin{tabular}{|c|c|c|c|c|c|c|c|c|c|}
\hline \multirow{2}{*}{ Análises urinárias } & \multicolumn{2}{|c|}{ Cirurgia intestinal } & \multirow{2}{*}{ Valor de $P^{*}$} & \multicolumn{2}{|c|}{ Ressecção ileal } & \multirow{2}{*}{ Valor de $P^{*}$} & \multicolumn{2}{|c|}{ Cálculo renal } & \multirow{2}{*}{ Valor de $P^{*}$} \\
\hline & $\operatorname{Sim}(19)$ & Não (10) & & $\operatorname{Sim}(11)$ & Não (18) & & $\operatorname{Sim}(13)$ & Não (16) & \\
\hline $\begin{array}{l}\text { Supersaturação relativa } \\
\text { de oxalato de cálcio }\end{array}$ & $\begin{array}{c}5,7 \pm 3,4 \\
(1,8-16,2)\end{array}$ & $\begin{array}{l}3,0 \pm 2,6 \\
(0,8-9,8)\end{array}$ & $0,007^{\dagger}$ & $\begin{array}{c}6,6 \pm 3,5 \\
(3,3-16,2)\end{array}$ & $\begin{array}{c}3,7 \pm 2,8 \\
(0,8-10,9)\end{array}$ & $0,007^{\dagger}$ & $\begin{array}{c}5,0 \pm 3,2 \\
(2,4-10,9)\end{array}$ & $\begin{array}{c}4,6 \pm 3,6 \\
(1,5-16,2)\end{array}$ & 0,693 \\
\hline $\begin{array}{l}\text { Supersaturação relativa } \\
\text { de brushita }\end{array}$ & $\begin{array}{c}1,1 \pm 0,8 \\
(0,1-2,6)\end{array}$ & $\begin{array}{c}0,4 \pm 0,3 \\
(0,1-0,9)\end{array}$ & $0,015^{\dagger}$ & $\begin{array}{c}1,3 \pm 0,9 \\
(0,2-2,6)\end{array}$ & $\begin{array}{c}0,6 \pm 0,6 \\
(0,1-2,5)\end{array}$ & $0,019^{\dagger}$ & $\begin{array}{c}08, \pm 0,8 \\
(0,2-2,4)\end{array}$ & $\begin{array}{c}0,9 \pm 0,8 \\
(0,1-2,6)\end{array}$ & 0,483 \\
\hline $\begin{array}{l}\text { Supersaturação relativa } \\
\text { de ácido úrico }\end{array}$ & $\begin{array}{r}1,9 \pm 1,4 \\
(0,1-4,5)\end{array}$ & $\begin{array}{c}2,4 \pm 2,1 \\
(0,2-5,8)\end{array}$ & 0,614 & $\begin{array}{c}1,7 \pm 1,2 \\
(0,2-3,7)\end{array}$ & $\begin{array}{l}2,3 \pm 1,9 \\
(0,1-5,8)\end{array}$ & 0,431 & $\begin{array}{c}1,8 \pm 1,5 \\
(0,2-4,8)\end{array}$ & $\begin{array}{c}2,3 \pm 1,8 \\
(0,1-5,8)\end{array}$ & 0,456 \\
\hline
\end{tabular}

* valor de $P$ usando teste $U$ de Mann-Whitney

$\dagger P<0,05$

são estratificados de acordo com o tipo de cirurgia, a excreção urinária de oxalato mostra-se, em média, maior naqueles com cirurgia ileal.

A oxalúria esteve, de maneira geral, dentro dos limites de normalidade, provavelmente pela ingestão deficiente de alimentos ricos em oxalato pela população brasileira (espinafre, ruibarbo, beterraba, nozes, acelga, amendoim, etc) ${ }^{(11)}$ ou pelo fato do estudo ter sido realizado em período de inatividade da doença.

A excreção urinária dos outros fatores promotores como o cálcio e o ácido úrico também não mostrou alteração significativa neste grupo de pacientes, entretanto, observou-se alta freqüência de hipocitratúria $(72,4 \%)$ e hipomagnesiúria $(48,3 \%)$. Considerando que tanto o citrato quanto o magnésio são importantes agentes inibidores da cristalização, suas deficiências podem favorecer o processo de crescimento e agregação dos cristais, principalmente dos sais de cálcio ${ }^{(2)}$.

Nesta série foi observada litíase renal em $44,8 \%$ dos pacientes, achado considerado alto. NISHIURA et al. ${ }^{(1)}$ observaram litíase renal em $25 \%$ dos pacientes com diagnóstico de doença inflamatória intestinal, SOTO et al. ${ }^{(15)}$ encontraram litíase renal em $16,3 \%$ e CAUDARELLA et al. ${ }^{(5)}$ encontraram freqüência de cálculos renais de $8,3 \%$ no pré-operatório e de $11,9 \%$ no pós-operatório dos pacientes submetidos a procedimento cirúrgico.
É descrito que a presença de supersaturação associada à insuficiência de inibidores da cristalização como magnésio, citrato e/ou outros fatores inibidores na urina, predispõe à formação de núcleos de cristalização ${ }^{(7,13)}$. Os pacientes deste estudo que haviam sido submetidos a intervenção cirúrgica intestinal mostraram possuir alterações significativas na saturação urinária e de acordo com ALVES et al. ${ }^{(2)}$, em todas as situações clínicas nas quais ocorre a supersaturação de oxalato de cálcio, observa-se aumento do risco de formação de litíase urinária.

Nos pacientes com cálculo renal, não se observam diferenças na excreção dos solutos e na saturação urinária quando comparados com indivíduos livres de cálculos urinários. Provavelmente esse resultado se deve ao número pequeno de pacientes da amostra. Entretanto, as alterações metabólicas encontradas em $93,1 \%$ deles, assim como a alta freqüência de hipocitratúria e de hipomagnesiúria e o alto número de pacientes com litíase urinária, mostram que esses pacientes possuem alterações significativas. Investigação em maior escala ou com duração mais longa e compreensão mais específica dos mecanismos determinantes da formação de cálculos renais neste grupo de pacientes, poderiam favorecer a atuação preventiva sobre os fatores que propiciam a formação do cálculo, diminuindo as complicações deles decorrentes. 
Viana MLL, Pontes RMA, Garcia WE, Fávero ME, Prete DC, Matsuo T. Crohn’s disease and kidney stones: much more than coincidence? Arq Gastroenterol. 2007;44(3):210-4.

ABSTRACT - Background - Crohn's disease is an inflammatory bowel disease associated with a wide variety of complications and manifestations secondary to the effects of underlie inflammatory process. In about $30 \%$ of the patients with Crohn's disease can be found extra-intestinals symptoms. Nephrolithiasis is one of them and the appearance of kidney stones, mainly of oxalate of calcium, is more common in these patients than in general population. Aim - To evaluate urinary metabolic factors potentially involved in renal stones formation on patients with Crohn's disease. Methods - We evaluated 29 patients with Crohn's disease followed in the Outpatient Bowel Inflammatory Disease Clinics of State University Hospital, Londrina, PR, Brazil, from January to December of 2004. The metabolic evaluation included measured of blood and urine substances related to renal stones formation, kidneys, ureters and bladder ultrassonography and calculation of urinary supersaturation for calcium oxalate, uric acid and calcium phosphate. Results - Twenty-nine of the evaluated patients were female or $65.5 \%$ and $34.5 \%$ were males. Among the metabolic urinary studied, we identified the following potential disturbances associated with nephrolithiasis: hypocitraturia in 21 patients (72.4\%), hypomagnesuria in 12 (41.4\%), hyperoxaluria in 4 (13.6\%) and urinary volume low in 5 (17.2\%). Renal stones were identified in 13 patients (44.8\%). Oxalate urinary excretion was higher in patients submitted to bowel surgery and also in patients with ileum resection. The urinary supersaturation of calcium oxalate and brushita in patients with bowels surgery was higher than the other patients not submitted to any surgery. Data to compare patients who underwent to surgery or not were analyzed by Mann-Whitney test ( $U$ test), and Qui-square test or the accurate test of Fisher have been used to determine variables association. For all tests $5 \%$ of significance level was considered. Conclusion - This study shows that the frequency of renal stones in patients suffering from Crohn's disease is higher than in general population and, also, higher than that described elsewhere for this disease. We identified hypocitraturia and hypomagnesiuria as the main factors which could explain our data. The impact of these metabolic disturbances can be also evaluated by means of the high supersaturation obtained for calcium oxalate and calcium phosphate which suggests the way those factors could be influencing crystal nucleation and, consequently, lead to renal stones formation in this group of patients.

HEADINGS - Crohn disease. Kidney calculi. Calcium oxalate.

\section{REFERÊNCIAS}

1. Ahlstrand C, Tiselius H. Urine composition and stone formation during treatment with acetazolamide. Scand J Urol Nephrol. 1987;21:225-8.

2. Alves R, Comiran C, Karbol C, Barros E. Ocorrência de litíase renal em pacientes com ressecção parcial do intestino delgado. J Bras Nefrol. 2000;22:55-61.

3. Bernades P, Heckestweiler P, Benozio M, Descos L, Geffroy Y, Hemet J, Loygue J, Modigliani R, Potet F, Weill JP. Proposition d'un système de critères pour le diagnostic des entérocolites inflammatoires cryptogénétiques (maladie de Crohn et rectocolite hémorragique). Gastroenterol Clin Biol. 1978;2:1047-54.

4. Best WR, Becktel JM, Singleton JW, Kern F. Development of a Crohn's disease activity index. National Cooperative Crohn's Disease Study. Gastroenterology. 1976;70:439-44.

5. Caudarella R, Rizzoli E, Pironi L, Malavolta N, Martelli G, Poggiolo G, Gozzetti G, Miglioli M. Renal stone formation in patients with inflammatory bowel disease. Scanning Microsc. 1993;7:371-9.

6. Caudarella R, Rizzoli E, Buffa A, Bottura A, Stefoni S. Comparative study of the influence of 3 types of mineral water in patients with idiopathic calcium lithiasis. J Urol. 1998;159:658-63.

7. Daudon M, Hennequin C, Bader C, Jungers P, Lacour B, Drüeck T. Inhibitors of crystallization. Adv Nephrol Necker Hosp. 1995;24:167-216.

8. Dowling RH. Rose GA, Sutor DJ. Hyperoxaluria and renal calculi in ileal disease. Lancet. 1971;1:1103-6.

9. Greenstein AJ, Janowitz HD, Sachar DB. The extra-intestinal complications of Crohn's disease and ulcerative colitis: a study of 700 patients. Medicine (Baltimore). 1976;55:401-12.
10. McConnell N, Campbell S, Gillander I, Rolton H, Danesh B. Risk factors for developing renal stones in inflammatory bowel disease. BJU Int. 2002;89:835-41.

11. Nishiura JL, Ferreira LC, Melo RP, Heilberg IP, Miszputen SJ, Schor N. Avaliação da saturação urinária em pacientes com doença inflamatória intestinal. J Bras Nefrol. 1994; 16:219-22.

12. Pardi DS, Tremaine WJ, Sandborn WJ, MsCarthy JT. Renal and urologic complications of inflammatory bowel disease. Am J Gastroenterol. 1998; 93:504-14.

13. Parks JH, Coward M, Coe FL. Correspondence between stone composition and urine supersaturation in nephrolithiasis. Kidney Int. 1997;51:894-900.

14. Ruml LA, Pearle MS, Pak CY. Medical therapy, calcium oxalate urolithiasis. Urol Clin North Am. 1997;24:117-33.

15. Soto AB, Jiménez RT, Oliveira A, Herraiz IFB, García AM, Antón FM. Lithogenic risk factors for renal stones in patients with Crohn's disease. Arch Esp Urol. 2001;54:282-92.

16. Sriboonlue P, Suwantrai S, Prasongwatana V. An indirect method for urinary oxalate estimation. Clin Chim Acta. 1998;273:59-68.

17. Tostes V, Cardoso LR. Recentes avanços em litíase urinária. J Bras Nefrol. 2001;23:166-73.

18. Valera JM, Morales A. Manifestaciones extraintestinales de la enfermedad de Crohn. Rev Hosp Clin Univ Chil. 2001;12:185-91.

19. Worcester EM. Stones from bowel disease. Endocrinol Metab Clin North Am. 2002;31:979-99. 